\title{
MOHAMMED-LARBI LABBI \\ Actions des groupes de Lie presque simples et positivité de la $p$-courbure
}

\author{
Annales de la faculté des sciences de Toulouse $6^{e}$ série, tome $6, \mathrm{n}^{\circ} 2$ \\ (1997), p. 263-276 \\ <http://www.numdam.org/item?id=AFST_1997_6_6_2_263_0>
}

(C) Université Paul Sabatier, 1997, tous droits réservés.

L'accès aux archives de la revue «Annales de la faculté des sciences de Toulouse » (http://picard.ups-tlse.fr/ annales/) implique l'accord avec les conditions générales d'utilisation (http://www.numdam.org/conditions). Toute utilisation commerciale ou impression systématique est constitutive d'une infraction pénale. Toute copie ou impression de ce fichier doit contenir la présente mention de copyright.

\section{Numdam}

Article numérisé dans le cadre du programme Numérisation de documents anciens mathématiques http://www.numdam.org/ 


\title{
Actions des groupes de Lie presque simples et positivité de la $p$-courbure ${ }^{(*)}$
}

\author{
Mohammed-Larbi LABBI ${ }^{(1)}$
}

RÉSuMÉ. - H. B. Lawson et S. T. Yau ont démontré dans [6] que si une variété compacte admet une action $C^{\infty}$ effective de $\mathrm{SU}(2)$ ou $\mathrm{SO}(3)$, alors elle admet une métrique riemannienne à courbure scalaire positive. En supposant que la variété admet une action d'un groupe de Lie compact, connexe, simple et de rang supérieur, on obtient une propriété de positivité plus forte.

Abstract - - H. B. Lawson and S. T. Yau proved in [6] that a compact manifold which admits a smooth and effective action of $\mathrm{SU}(2)$ or $\mathrm{SO}(3)$ carries a riemannian metric of positive scalar curvature. Supposing the manifold admits an action of a compact, connected, simple Lie group of higher rank, we get a stronger positivity property.

\section{Introduction}

La $p$-courbure d'une variété riemannienne $(M, g)$, notée $s_{p}(p=0, \ldots$, $n-2)$, est une fonction définie sur le fibré en $p$-grassmanniennes de $M$ comme suit.

Si $P$ est un $p$-plan dans $T_{m} M(m \in M), s_{p}(P)$ est la courbure scalaire au point $m$ de la sous-variété riemannienne $\exp _{m}(V)$ de $(M, g)$ où $V$ est un voisinage de 0 dans $P^{\perp}$ (l'orthogonal à $P$ dans $T_{m} M$ ).

(*) Reçu le $1^{\text {er }}$ juillet 1994

(1) C.N.R.S. Unité Associée 1407, Département de Mathématiques, case 51, Université Montpellier II, F-34095 Montpellier Cedex 5 (France) 
Autrement dit

$$
s_{p}(P)=\sum_{j, k=p+1}^{n} K_{m}\left(e_{j}, e_{k}\right)
$$

où $\left(e_{p+1}, \ldots, e_{n}\right)$ est une base orthonormée quelconque de $P^{\perp}$, et $K$ désigne la courbure sectionnelle de $(M, g)$.

Pour $p=0$ (resp. $p=n-2$ ) on retrouve la courbure scalaire (resp. la courbure sectionnelle) de $(M, g)$. De plus, la 1-courbure $(p=1)$ coïncide (à un facteur 2 près) avec la forme quadratique associée au tenseur d'Einstein $\left(s_{0} / 2\right) g-$ Ric, où Ric désigne la courbure de Ricci de $(M, g)$.

\section{Exemples}

1) Pour une variété riemannienne de dimension $n$ à courbure sectionnelle constante $k$, on a

$$
s_{p}=(n-p)(n-p-1) k
$$

2) Pour une variété d'Einstein de dimension $n$ et de constante d'Einstein $r=s_{0} / n$, on a

$$
s_{1}(n-1) r \quad \text { et } \quad s_{2}(P)=(n-4) r+2 K(P) .
$$

Remarque. - Cette notion de $p$-courbure n'est pas la même que celle introduite par J. A. Thorpe dans [9].

Il est facile de vérifier que si $\left(e_{p+1}, \ldots, e_{n}\right)$ est une base orthonormée de $P^{\perp}$, alors

$$
\sum_{k=p+1}^{n} s_{p+1}\left(\left\langle P, e_{k}\right\rangle\right)=(n-p-2) s_{p}(P) .
$$

D'où, $s_{p+1}>0 \Rightarrow s_{p}>0$; en particulier, la positivité de la $p$-courbure entraîne la positivité de la courbure scalaire.

On se propose alors de généraliser les propriétés de positivité de la courbure scalaire à la $p$-courbure. Dans ce sens, on a montré dans [4] et [5] les deux résultats suivants :

a) si $(M, g)$ est une variété compacte conformément plate de dimension $n$ à $p$-courbure positive, alors

$$
H^{m}(M, \mathbb{R})=0 \quad \text { pour } \quad \frac{n-p}{2} \leq m \leq \frac{n+p}{2}
$$


Actions des groupes de Lie presque simples et positivité de la $p$-courbure

b) si $(M, g)$ est une variété riemannienne compacte à $p$-courbure positive, alors toute variété obtenue à partir de $M$ par chirurgie de codimension $\geq p+3$ admet une métrique riemannienne à $p$-courbure positive.

Dans cet article, on montre le résultat suivant.

THÉORÈME PRINCIPAL .- Si une variété compacte $M$ admet une action $C^{\infty}$ (non triviale) d'un groupe de Lie $G$ compact, connexe, presque simple et de rang supérieur ou égal à $p+1$; alors elle admet une métrique riemannienne à p-courbure positive.

\section{Submersions riemanniennes et $p$-courbure}

\subsection{Variation canonique}

Soient $(M, g)$ et $(B, \check{g})$ deux variétés riemanniennes, $\pi:(M, g) \rightarrow(B, \check{g})$ une submersion riemannienne; on définit pour tout $t \in \mathbb{R}$ une nouvelle métrique $g_{t}$ sur $M$ en multipliant $g$ par $t^{2}$ dans les directions tangentes aux fibres. Évidemment, $\pi:\left(M, g_{t}\right) \rightarrow(B, \check{g})$ est encore une submersion riemannienne avec la même distribution horizontale et verticale, voir [1].

\section{Notations}

- On indexe par $t$ les invariants de la métrique $g_{t}$, dans le cas $t=1$ on ne met pas l'indice.

- On met sous chapeau “へ” (resp. sous “ " ”) les invariants de la fibre (resp. de la base $B$ ).

- $U, V, W, W^{\prime}$ désignent des champs de vecteurs verticaux sur $M$.

- $X, Y, Z, Z^{\prime}$ désignent des champs de vecteurs horizontaux sur $M$.

- $R$ est le tenseur de courbure de Riemann de $\left(M, g_{t}\right)$ pour $t=1$.

- $\widehat{R}$ désigne la collection des tenseurs de courbure de Riemann des fibres munis de la métrique induite par $g_{t}$ pour $t=1$.

En utilisant à la fois les formules d'O'Neill pour la courbure [8] et le lemme 9.69 de [1, p. 252], on aura le résultat suivant. 
LEMME 2.1

$$
\begin{aligned}
R_{t}\left(U, V, W, W^{\prime}\right)= & t^{2} \widehat{R}\left(U, V, W, W^{\prime}\right)-t^{4} g\left(T_{U} W, T_{V} W^{\prime}\right)+ \\
& +t^{4} g\left(T_{V} W, T_{U} W^{\prime}\right) \\
R_{t}(U, V, W, X)= & t^{2} R(U, V, W, X)+ \\
& +t^{2}\left(1-t^{2}\right)\left\{g\left(T_{U} W, A_{X} V\right)-g\left(T_{V} W, A_{X} U\right)\right\} \\
R_{t}(X, U, Y, V)= & t^{2} R(X, U, Y, V)-t^{2}\left(1-t^{2}\right) g\left(A_{X} V, A_{Y} U\right) \\
R_{t}(U, V, X, Y)= & t^{2} R(U, V, X, Y)+ \\
& +t^{2}\left(1-t^{2}\right)\left\{g\left(A_{X} U, A_{Y} V\right)-g\left(A_{X} V, A_{Y} U\right)\right\} \\
R_{t}(X, Y, Z, U)= & t^{2} R(X, Y, Z, U) \\
R_{t}\left(X, Y, Z, Z^{\prime}\right)= & R\left(X, Y, Z, Z^{\prime}\right)+\left(1-t^{2}\right)\left\{2 g\left(A_{X} Y, A_{Z} Z^{\prime}\right)+\right. \\
& \left.-g\left(A_{Y} Z, A_{X} Z^{\prime}\right)+g\left(A_{X} Z, A_{Y} Z^{\prime}\right)\right\}
\end{aligned}
$$

où

$$
\begin{aligned}
& T_{E_{1}} E_{2}=\mathcal{H} D_{\mathcal{V} E_{1}} \mathcal{V} E_{2}+\mathcal{V} D_{\mathcal{V} E_{1}} \mathcal{H} E_{2} \\
& A_{E_{1}} E_{2}=\mathcal{H} D_{\mathcal{H} E_{1}} \mathcal{V} E_{2}+\mathcal{V} D_{\mathcal{H} E_{1}} \mathcal{H} E_{2}
\end{aligned}
$$

on a désigné par $\mathcal{H} E$ (resp. $\mathcal{V} E$ ) la composante horizontale (resp. verticale) de $E$.

Dans la suite, $E_{i}$ désignera un vecteur tangent à $M$ de la forme

$$
E_{i}=\alpha_{i} U_{i}+\beta_{i} X_{i} \text { avec }\left\|U_{i}\right\|_{t}=\left\|X_{i}\right\|_{t}=1 \text { et } \alpha_{i}^{2}+\beta_{i}^{2}=1 .
$$

D'après le lemme précédent on a

$$
K_{t}\left(E_{1}, E_{2}\right)=t^{2} \widehat{R}\left(\alpha_{1} U_{1}, \alpha_{2} U_{2}, \alpha_{1} U_{1}, \alpha_{2} U_{2}\right)+\alpha_{1} \alpha_{2} \mathrm{O}\left(\frac{1}{t}\right)+\mathrm{O}(1)
$$

Si $P$ est un 2-plan dans $T_{m} M, m \in M$, et si $\mathcal{H}_{m}$ (resp. $\mathcal{V}_{m}$ ) est la partie verticale (resp. horizontale) de la submersion $\pi$, deux cas sont possibles :

i) $P \cap \mathcal{H}_{m} \neq\{0\}$ alors, d'après la formule (2), on a $K_{t}(P)=\mathrm{O}(1)$;

ii) $P \cap \mathcal{H}_{m}=\{0\}$, soit alors $\left(E_{1}, E_{2}\right)$ une base orthonormée de $P$ telle que sa projection orthogonale $\left(U_{1}, U_{2}\right)$ sur $\mathcal{V}_{m}$ soit orthogonale (ce qui 
Actions des groupes de Lie presque simples et positivité de la $p$-courbure

revient à diagonaliser simultanément deux produits scalaires). Dans ce cas, la formule (2) s'écrit

$$
K_{t}(P)=K_{t}\left(E_{1}, E_{2}\right)=\frac{\alpha_{1}^{2} \alpha_{2}^{2}}{t^{2}} \widehat{K}\left(U_{1}, U_{2}\right)+\mathrm{O}\left(\frac{1}{t}\right) .
$$

Enfin, soit $P^{\perp}$ l'orthogonal à un $p$-plan $P$ dans $T_{m} M$, et $q=\operatorname{dim} \mathcal{V}_{m}$, alors il est facile de vérifier que

$$
\operatorname{dim}\left(P^{\perp} \cap \mathcal{V}_{m}\right) \geq q-p .
$$

D'où, il existe au moins $q-p$ vecteurs verticaux $U_{1}, \ldots, U_{q-p}$ dans $P^{\perp}$. Ainsi, en supposant $\widehat{K} \geq 0$, on a

$$
\left(s_{p}\right)_{t}(P)=\frac{1}{t^{2}} \sum_{1 \leq i, j \leq q-p} \widehat{K}\left(U_{i}, U_{j}\right)+(\text { termes } \geq 0)+\mathrm{O}\left(\frac{1}{t}\right) .
$$

On remarque que le premier terme est une $p$-courbure de la fibre munie de la métrique induite. Ainsi on a montré le résultat suivant.

THÉORÈME 2.2.- Si les fibres de $\pi:(M, g) \rightarrow(B, \check{g})$ sont à courbure sectionnelle non négative et à p-courbure positive, alors $\forall m \in M$ et $\forall P$ un p-plan de $T_{m} M, \exists t_{0}$ tel que $\forall t \leq t_{0},\left(s_{p}\right)_{t}(P)>0$.

En particulier si $M$ est compacte, $\exists t_{0}$ tel que $\forall t \leq t_{0},\left(M, g_{t}\right)$ est à p-courbure positive.

Remarque. - Cette technique de variation canonique de la métrique de l'espace total d'une submersion riemannienne pour faire apparaître de la courbure positive a été utilisée par de nombreux auteurs. Citons par exemple, J. Cheeger dans [2], H. B. Lawson et S. T. Yau dans [6] et A. L. Besse dans [1].

Corollaire 1. - Le produit d'une variété compacte arbitraire avec la sphère $S^{m}, m \geq p+2$, admet une métrique riemannienne à $p$-courbure positive.

Preuve. - C'est une conséquence directe du théorème $2.2 \square$

Corollaire 2.- Si une variété compacte admet un feuilletage riemannien dont les feuilles sont à courbure sectionnelle non négative et à $p$ courbure positive, alors elle admet une métrique riemannienne à p-courbure positive. 
Preuve. - Dans ce cas, on a localement une submersion riemannenne et il suffit de remarquer que le théorème 2.2 est encore vrai dans ce cas.

Remarque. - La condition de positivité de la $p$-courbure des fibres dans le théorème 2.2 et le corollaire 2 implique que $p \leq \operatorname{dim}(\mathrm{fibre})-2$.

Corollaire 3.- Si une variété compacte $M$ admet une action libre d'un groupe de Lie $G$ compact, connexe, presque simple et de rang $r$, alors elle admet une métrique riemannienne à p-courbure positive pour $p \leq r(r+1)-1$.

Preuve. - La projection canonique $M \rightarrow M / G$ est dans ce cas une submersion lisse. On munit les fibres de la métrique bi-invariante de $G$ via l'inclusion canonique $\mathcal{G} \hookrightarrow T_{m} M$. À partir d'une métrique $G$-invariante sur $M$, on définit la distribution horizontale sur laquelle on relève une métrique quelconque de la base $M / G$, ainsi $M \rightarrow M / G$ est une submersion riemannienne.

D'autre part, $G$ muni de sa métrique bi-invariante est à $p$-courbure positive s'il existe dans l'orthogonal de tout $p$-plan dans $\mathcal{G}$ au moins un 2-plan à courbure sectionnelle positive, autrement $\operatorname{dit} \operatorname{sid} \operatorname{dim} G-\operatorname{rang} G \geq$ $p+1$. En effet, il est bien connu que la courbure sectionnelle d'un groupe de Lie muni d'une métrique bi-invariante s'écrit :

$$
K(X, Y)=\frac{1}{4}\|[X, Y]\|^{2} .
$$

En particulier, $K(X, Y) \geq 0$; de plus, $K(X, Y)=0$ si et seulement si $[X, Y]=0$.

Or, d'après la classification des groupes de Lie compacts, connexes et presque simples (voir [3] ou tableau du lemme 3.2) on a $\operatorname{dim} G-r \geq r(r+1)$ où $r$ est le rang de $G$. Le corollaire résulte alors à partir du théorème 2.2 .

\section{Actions des groupes de Lie et $p$-courbure}

Soit $M$ une variété admettant une action $C^{\infty}$ d'un groupe de Lie $G$ compact. Notons par $b$ une métrique bi-invariante sur $G$ et par $g_{0}$ une métrique $G$-invariante quelconque sur $M$. 
Actions des groupes de Lie presque simples et positivité de la $p$-courbure

\subsection{Préambule}

Dans ce paragraphe, on construit (comme dans [6]) à partir de l'action de $G$, une nouvelle métrique sur $M$ comme suit : soit $G \times M$ muni de la métrique produit $b \times g_{0}$ et soit $\Phi$ l'action diagonale de $G$ sur $G \times M$ définie par $\Phi_{g}(h, x)=(g \cdot h, g \cdot x)$. C'est une action libre.

Soit $\pi^{\Phi}$ la projection canonique $G \times M \rightarrow M$. Elle est donnée par $\pi^{\Phi}(g, x)=g^{-1} \cdot x$. On définit une deuxième métrique $g$ sur $G \times M$ pour laquelle $\pi^{\Phi}$ est une submersion riemannienne :

- le long des orbites on introduit la métrique bi-invariante $b$ au moyen de la $\Phi$-action libre de $G$ sur $G \times M$;

- en utilisant la métrique produit $b \times g_{0}$, on définit un champ de plans normaux aux orbites de la $\Phi$-action, sur lesquels on relève la métrique $g_{0}$ de $M$; soit $g_{t}$ la variation canonique de $g, 0<t \leq 1$.

On remarque que, pour tout $t$, la métrique $g_{t}$ sur $G \times M$ est invariante sous la $\psi$-action de $G$ donnée par $\psi_{g}(h, x)=\left(h \cdot g^{-1}, x\right)$; et donc il existe une métrique $\tilde{g}_{t}$ sur $M$ pour laquelle la projection à droite $\pi: G \times M \rightarrow M$ est une submersion riemannienne.

\section{Notations}

- $\mathcal{V}_{m}, \mathcal{H}_{m}$ (resp. $\mathcal{V}_{m}^{\prime}, \mathcal{H}_{m}^{\prime}(t)$ ) désignent les parties verticales et horizontales pour la submersion riemannienne $\pi^{\Phi}$ (resp. $\pi$ ) au point $(e, m) \in G \times M, e$ étant l'élément neutre de $G$.

- $\mathcal{G}=\mathcal{G}_{m} \oplus \mathcal{P}_{m}$ l'algèbre de Lie de $G$ où $\mathcal{G}_{m}$ est l'algèbre de Lie du sousgroupe d'isotropie $G_{m}$ au point $m, \mathcal{P}_{m}$ est le supplémentaire orthogonal de $\mathcal{G}_{m}$ pour la métrique bi-invariante sur $G$.

- $i_{m}$ désigne l'injection naturelle $\mathcal{P}_{m} \hookrightarrow T_{m} M$ donnée par l'action de $G$ sur $M$.

- $t_{m}$ désigne le supplémentaire orthogonal de $i_{m} \mathcal{P}_{m}$ dans $T_{m} M$ pour $g$. Ainsi, on a une décomposition orthogonale de $T_{(e, m)}(G \times M)$ pour la métrique produit

$$
T_{(e, m)}(G \times M)=\mathcal{G}_{m} \oplus \mathcal{P}_{m} \oplus i_{m} \mathcal{P}_{m} \oplus t_{m}
$$

Proposition 3.1. - Pour tout $m \in M$ et pour tout $t$, on a

$$
\mathcal{H}_{m}^{\prime}(t) \cap \mathcal{H}_{m}=t_{m}, \quad g_{t}\left(\mathcal{H}_{m}^{\prime}(t), \mathcal{G}_{m}\right)=0
$$


Preuve. - L'injection naturelle $j_{m}: \mathcal{G} \hookrightarrow T_{(e, m)}(G \times M)$ est donnée par

$$
\begin{aligned}
\mathcal{G}=\mathcal{G}_{m} \oplus \mathcal{P}_{m} & \rightarrow T_{(e, m)}(G \times M)=\mathcal{G}_{m} \oplus \mathcal{P}_{m} \oplus i_{m} \mathcal{P}_{m} \oplus t_{m} \\
(u, v) & \rightarrow\left(u, v, i_{m} v, 0\right)=(u, 0,0,0)+\left(0, v, i_{m} v, 0\right)
\end{aligned}
$$

D'où la partie verticale pour $\pi^{\Phi}$ est

$$
\mathcal{V}_{m}=j_{m}(\mathcal{G})=\mathcal{G}_{m} \oplus j_{m} \mathcal{P}_{m}
$$

De plus

$$
j_{m} \mathcal{P}_{m} \cap \mathcal{P}_{m}=\{0\} \quad \text { et } \quad j_{m} \mathcal{P}_{m} \cap i_{m} \mathcal{P}_{m}=\{0\}
$$

Remarque. - On voit que les sous-espaces $\mathcal{G}_{m}$ et $t_{m}$ sont orthogonaux au sous-espace $\mathcal{P}_{m}+i_{m} \mathcal{P}_{m}$ à la fois pour la métrique $g_{t}$ et pour la métrique produit.

Soient $H_{m}$ et $H_{m}^{\prime}(t)$ les deux sous-espaces de $\mathcal{P}_{m}+i_{m} \mathcal{P}_{m}$ définis par

$$
\mathcal{P}_{m}+i_{m} \mathcal{P}_{m}=j_{m} \mathcal{P}_{m} \oplus_{t} H_{m}=\mathcal{P}_{m} \oplus_{t} H_{m}^{\prime}(t)
$$

où $\bigoplus_{t}$ est la somme directe pour le produit scalaire $g_{t}$ sur $T_{(e, m)}(G \times M)$.

Par définition $H_{m}$ est orthogonal à $\mathcal{V}_{m}=\mathcal{G}_{m} \bigoplus_{t} j_{m} \mathcal{P}_{m}$ pour la métrique $g_{t}$ donc aussi pour la métrique produit (par définition de $g_{t}$ ), alors en utilisant (3) on a

$$
H_{m} \cap \mathcal{P}_{m}=\{0\} \quad \text { et } \quad H_{m} \cap i_{m} \mathcal{P}_{m}=\{0\}
$$

Par conséquent

$$
H_{m}^{\prime}(t) \cap H_{m}=\mathcal{P}_{m}^{\perp} \cap j_{m} \mathcal{P}_{m}^{\perp}=\left(\mathcal{P}_{m}+j_{m} \mathcal{P}_{m}\right)^{\perp}=\{0\}
$$

où $\perp$ est pris dans le sous-espace $\mathcal{P}_{m}+i_{m} \mathcal{P}_{m}$ pour la métrique $g_{t}$. Et on conclut en remarquant que

$$
\mathcal{H}_{m}^{\prime}(t)=H_{m}^{\prime}(t) \oplus_{t} t_{m} \quad \text { et } \quad \mathcal{H}_{m}=H_{m} \oplus_{t} t_{m} \cdot \square
$$


Actions des groupes de Lie presque simples et positivité de la $p$-courbure

\subsection{Contrôle de la $p$-courbure en dehors de l'ensemble des points} fixes

Dans ce paragraphe on démontre le théorème suivant.

THÉORÈME 3.2.- Soit G un groupe de Lie compact, connexe, presque simple, et de rang au moins $p+1$, alors, quel que soit le voisinage $U$ de l'ensemble des points fixes de l'action de $G$ sur $M$, il existe $t_{0}$ tel que $\forall t \leq t_{0}$ la métrique $\tilde{g}_{t}$ est à p-courbure positive sur $M \backslash U$.

La démonstration utilise les ingrédients donnés ci-dessous.

DÉfinition .- Soit $\mathcal{D}_{m} \subset T_{m} M$ un q-plan, $P \subset \mathcal{D}_{m}$ un p-plan. On appellera p-courbure de $\mathcal{D}_{m}$ en $P$ la somme

$$
\sum_{i, j=1}^{q-p} K\left(e_{i}, e_{j}\right)
$$

où $\left(e_{1}, \ldots, e_{q-p}\right)$ est une base orthonormée de $P^{\perp} \cap \mathcal{D}_{m}$ et $K$ désigne la courbure sectionnelle de $(M, g)$.

Remarque. - Soit $\pi:(M, g) \rightarrow(B, \check{g})$ une submersion riemannienne et $\mathcal{D}_{m} \subset T_{m} M$. D'après la démonstration du théorème 2.2 (en reprenant les mêmes notations) on a : pour tout $m \in M$, si la projection orthogonale de $\mathcal{D}_{m}$ sur la partie verticale $\mathcal{V}_{m}$ est à $p$-courbure positive et à courbure sectionnelle non négative, il existe alors $t_{0}$ tel que $\forall t \leq t_{0}\left(\mathcal{D}_{m}, g_{t}\right)$ est à $p$-courbure positive.

Lemme 3.3.- Soient $G$ comme dans le théorème 3.2 muni de sa métrique bi-invariante, $H$ un sous-groupe fermé quelconque de $G, \mathcal{G}$ (resp.

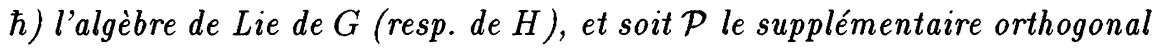
de $\hbar$ dans $\mathcal{G}$. Si $\mathcal{P} \neq\{0\}$ (i.e. si $H \neq G$ ), alors $\mathcal{P}$ est à p-courbure positive (au sens de la définition précédente).

Preuve. - Rappelons que la courbure sectionnelle de $G$ pour la métrique bi-invariante s'écrit

$$
K(X, Y)=\frac{1}{4}\|[X, Y]\|^{2} .
$$

En particulier $K(X, Y) \geq 0$. De plus, $K(X, Y)=0$ si et seulement si $[X, Y]=0$. 
Appelons rang $\mathcal{P}$ la dimension maximale des sous-espaces plats de $\mathcal{P}$, c'est-à-dire les sous-espaces de $\mathcal{P}$ sur lesquels la courbure sectionnelle (pour la métrique bi-invariante sur $G$ ) est partout nulle. On a évidemment $\operatorname{rang} \mathcal{P} \leq \operatorname{rang} G$. Alors $\mathcal{P}$ est à $p$-courbure positive dès que

$$
\operatorname{dim} \mathcal{P}-\operatorname{rang} \mathcal{P} \geq p+1
$$

D'autre part, on sait déterminer les sous-groupes propres connexes de dimension maximale de $G$ (voir [7]) donc la dimension minimale de $\mathcal{P}$. Notons par $H_{M}$ le sous-groupe connexe de dimension maximale de $G$ et par $\mathcal{P}_{M}$ le supplémentaire orthogonal à $\hbar_{M}$; d'après [7], on a :

\begin{tabular}{|l|c|c|l|c|}
\hline type $G$ & $\operatorname{dim} G$ & $\operatorname{dim} H_{M}$ & type $H_{M}$ & $\operatorname{dim} \mathcal{P}_{M}$ \\
\hline$A_{r}, r \geq 1$ & $r(r+2)$ & $r^{2}$ & $A_{r-1} \oplus S^{1}$ & $2 r$ \\
$B_{r}, r \geq 2$ & $r(2 r+1)$ & $r(2 r-1)$ & $D_{r}$ & $2 r$ \\
$C_{r}, r \geq 3$ & $r(2 r+2)$ & $(r-1)(2 r-1)+3$ & $C_{r-1} \oplus B_{1}$ & $4(r-1)$ \\
$D_{r}, r \geq 4$ & $r(2 r-1)$ & $(r-1)(2 r-1)$ & $B_{r-1}$ & $2(r-1)$ \\
$G_{2}$ & 14 & 8 & $A_{2}$ & 6 \\
$F_{4}$ & 52 & 36 & $B_{4}$ & 16 \\
$E_{6}$ & 78 & 52 & $F_{4}$ & 26 \\
$E_{7}$ & 133 & 79 & $E_{6} \oplus S^{1}$ & 54 \\
$E_{8}$ & 248 & 136 & $E_{7} \oplus B_{1}$ & 112 \\
\hline
\end{tabular}

Ainsi, $\operatorname{dim} \mathcal{P} \geq 2 r, r=\operatorname{rang} G$, sauf pour $D_{r}$. Or,

$$
\operatorname{dim} \mathcal{P} \geq 2 r \Rightarrow \operatorname{dim} \mathcal{P}-\operatorname{rang} G \geq r ;
$$

d'où $r \geq p+1 \Rightarrow(4)$ est satisfaite. Dans le cas de $D_{r}$, on a $\operatorname{dim} \mathcal{P} \geq 2 r-1$ avec égalité dans le cas où $H_{M}$ est du type $B_{r-1}$. Dans ce cas rang $\mathcal{P}=1$ et alors la condition (4) est satisfaite si $r \geq(p+3) / 2$.

Démonstration du théorème 3.2

Soit $m \in M$ non fixé par l'action de $G$ sur $M$.

D'une part, d'après les formules d'O'Neill pour la courbure, les courbures sectionnelles dans les directions horizontales augmentent si on passe à la base (voir [8]), et par conséquent $\left(T_{m} M, \widetilde{g}_{t}\right)$ est à $p$-courbure positive dès que $\left(\mathcal{H}_{m}^{\prime}(t), g_{t}\right)$ l'est. 
Actions des groupes de Lie presque simples et positivité de la $p$-courbure

D'autre part, d'après le lemme ci-dessus, si rang $G \geq p+1$ alors $j_{m} \mathcal{P}_{m}$ muni de la métrique induite par $g$ (qui est par construction la restriction à $j_{m} \mathcal{P}_{m}$ de la métrique bi-invariante sur $\mathcal{V}_{m}$ ) est à $p$-courbure positive (pourvu que $j_{m} \mathcal{P}_{m} \neq\{0\}$, c'est-à-dire pourvu que $m$ ne soit pas un point fixe de l'action de $G$ sur $M$ ).

Enfin, d'après la proposition 3.1, la projection orthogonale de $\mathcal{H}_{m}^{\prime}(t)$ sur $\mathcal{V}_{m}$ n'est autre que $j_{m} \mathcal{P}_{m}$. Le théorème résulte alors à partir de la remarque ci-dessus.

\subsection{Contrôle de la $p$-courbure au voisinage de l'ensemble des points fixes}

Soient $m \in M$ un point fixe sous l'action de $G$ sur $M$. Dans ce cas, la partie horizontale et verticale pour $\pi$ et $\pi^{\Phi}$ coïncident au point $(e, m)$. Notons par $K_{0}$ (resp. $K_{t}, \widetilde{K}_{t}$ ) la courbure sectionnelle de la métrique de départ $g_{0}$ sur $M$ (resp. $g_{t}$ sur $G \times M, \tilde{g}_{t}$ sur $M$ ), et par $\widetilde{s}_{p}^{t}$ (resp. $s_{p}^{0}$ ) la $p$-courbure de la métrique $\tilde{g}_{t}$ (resp. $g_{0}$ ).

D'après le lemme 2.1 on a

$$
K_{t}(X, Y)=K_{0}(X, Y)-3 t^{2}\left\|A_{X} Y\right\|^{2}
$$

où $\|\cdot\|$ est la norme associée à la métrique $g_{t}$ pour $t=1$. D'autre part, $\widetilde{K}_{t}(X, Y) \geq K_{t}(X, Y)$, et par conséquent si $P$ est un $p$-plan dans $T_{m} M$ on a

$$
\tilde{s}_{p}^{t}(P) \geq \widetilde{s}_{p}^{0}(P)+\mathrm{O}\left(t^{2}\right) .
$$

D'où, pour $t$ assez petit, la métrique $\tilde{g}_{t}$ est à $p$-courbure positive sur l'ensemble des points fixes dès que la métrique $g_{0}$ l'est.

Proposition 3.4. - Si rang $G \geq p+1$, alors $M$ admet une métrique riemannienne G-invariante à $p$-courbure positive au voisinage de l'ensemble des points fixes.

Preuve. - Rappelons que l'ensemble des points fixes est une réunion finie de sous-variétés de $M$.

Soient $K$ une composante connexe de l'ensemble des points fixes de codimension $k, h$ une métrique $G$-invariante quelconque sur $M$ et $N K$ le fibré normal de $K$ dans $M$. Le groupe $G$ agit linéairement sur $N K$; sur ce dernier on définit une métrique $G$-invariante $g^{\prime}$ comme suit : sur les fibres de $N K \rightarrow K$, on introduit la métrique à courbure sectionnelle constante $c>0$; 
précisément, les fibres sont difféomorphes à $\mathbb{R}^{k}$ sur laquelle on transporte par la projection stéréographique la métrique à courbure constante $c>0$ de la sphère $S^{k}$.

En utilisant la connexion normale sur $N K$ (définie à partir de la métrique $h$ ), on relève aux espaces horizontaux la métrique induite sur $K$ par $h$.

La métrique $g^{\prime}$ ainsi obtenue sur $N K$ est $G$-invariante, et la projection naturelle $\left(N K, g^{\prime}\right) \rightarrow(K, h)$ est une submersion riemannienne.

De plus, les fibres de cette submersion sont à $p$-courbure positive, en effet :

- d'une part puisque les fibres sont à courbure sectionnelle positive, il suffit que $k-2 \geq p$

- d'autre part d'après la démonstration du lemme 3.3 , on a rang $G \geq$ $p+1 \Rightarrow \operatorname{dim} \mathcal{P}_{x} \geq p+2 \forall x$ tel que $\mathcal{P}_{x} \neq\{0\}\left(\mathcal{P}_{x}\right.$ est orthogonal à $\left.\mathcal{G}_{x}\right) ;$

- de plus pour des points $x$ proches de $K$, on a $k \geq \operatorname{dim} \mathcal{P}_{x} ;$ d'où $k \geq p+2$.

Ce qui démontre l'affirmation.

Soit $g_{\varepsilon}^{\prime}$ la variation canonique de $g^{\prime}$ sur $N K$. Alors, d'après le théorème 2.2 ; pour tout $(m, n)$ dans $N K$ et tout $p$-plan dans $T_{(m, n)} N K$, il existe $\varepsilon_{0}$, tel que $\forall \varepsilon \leq \varepsilon_{0}\left(T_{(m, n)} N K, g_{\varepsilon}^{\prime}\right)$ est à $p$-courbure positive. La section nulle dans $N K$, identifiée à $K$, est compacte; il existe alors $\varepsilon_{0}$ tel que $\forall \varepsilon \leq \varepsilon_{0}$ la métrique $g_{\varepsilon}^{\prime}$ est à $p$-courbure positive sur $K$. Par continuité, il existe un voisinage de $K$ dans $N K$ qui est à $p$-courbure positive $\forall \varepsilon \leq \varepsilon_{0}$. Et, par équivariance de l'application exp $: N K \rightarrow M$, il existe un voisinage de $K$ dans $M$ qui est à $p$-courbure positive pour la métrique $\exp _{*} g_{\varepsilon}^{\prime}$. Cette métrique est $G$-invariante car $g_{\varepsilon}^{\prime}$ l'est par construction.

Ensuite, on prolonge à $M$, la métrique obtenue au voisinage de $K$, sans la changer dans un voisinage de $K$. Enfin, chaque élément de $G$ agit sur la métrique ainsi obtenue par image réciproque. On considère la métrique $G$-invariante obtenue en prenant la moyenne de ces actions par rapport à la mesure de Haar (cette opération ne modifie pas la métrique initiale sur un voisinage de $K$ car $G$-invariante par construction). Ce qui achève la démonstration de la proposition 3.4. 
Actions des groupes de Lie presque simples et positivité de la $p$-courbure

\section{Démonstration du théorème principal}

On prend comme métrique de départ sur $M$ la métrique $g_{0}$ donnée par la proposition 3.4. D'après la formule (5), il existe alors $t_{0}$ tel que $\forall t \leq t_{0}$ la métrique $\tilde{g}_{t}$ est à $p$-courbure positive sur l'ensemble des points fixes, par continuité, cela reste vrai dans un voisinage. Et on conclut en utilisant le théorème 3.2 .

\section{Remarques}

Il résulte de ce travail, que dans le cas d'une action effective d'un groupe de Lie non abélien, compact et connexe, la condition suivante sur $G$ est suffisante pour l'existence d'une métrique à $p$-courbure positive sur $M$. Pour tout sous-groupe fermé $H$ de $G$, différent de $G$, on a

$$
\operatorname{dim} G / H-\operatorname{rang} G \geq p+1 .
$$

Par conséquent, dans chacun des cas suivants, on peut améliorer les hypothèses du théorème principal supposées sur $G$.

- Dans le cas $p=0$, il n'est plus nécessaire de supposer que le groupe $G$ est presque simple; en effet, on peut se restreindre au cas où $G$ est $\mathrm{SU}(2)$ ou $\mathrm{SO}(3)$ (car il contient nécessairement l'un d'eux, voir [3]); dans ce cas, on a $\operatorname{dim} G / H \geq 2$ et $\operatorname{rang} G=1$; ainsi, on retrouve le théorème de H. B. Lawson et S. T. Yau;

Notre façon de contrôler la courbure (scalaire) au voisinage des points fixes est toutefois plus simple. Cette simplification était connue de L. BérardBergery (manuscrit non publié), comme me l'a signalé le referee.

- Dans le cas où $G$ est presque simple, selon le type de $G$, on peut affaiblir les hypothèses faites sur le rang du groupe $G$ (tableau, $\S 3.2$ ).

\section{Remerciements}

Je remercie J. Lafontaine pour des conversations utiles sur le sujet, ainsi que le referee pour sa lecture approfondie d'une première version de cet article. 


\section{Bibliographie}

[1] Besse (A. L.) .- Einstein manifolds, Springer-Verlag (1987).

[2] Cheeger (J.) .- Some examples of manifolds of non negative curvature, J. Differential Geometry 8 (1973), pp. 623-628.

[3] Dieudonné (J.) . - Fondements de l'analyse moderne, tome 5, Gauthier-Villars, Paris (1963).

[4] LABBi (M.-L.) .- Sur les nombres de Betti des variétés conformément plates, C.R. Acad. Sci. Paris, série I, 319 (1994), pp. 77-80.

[5] LABBi (M.-L.) .- Chirurgies et positivité de la p-courbure, Prépublication du Dép. de Mathématiques de l'Université Montpellier II.

[6] LaWson (H. B.) et YAU (S. T.) .- Scalar curvature, non abelian group actions, and the degree of symmetry of exotic spheres, Comment. Math. Helv. 49 (1974), pp. 232-244.

[7] MANN (L. N.) .- Gaps in the dimensions of transformation groups, Illinois J. Math. 10 (1966), pp. 532-546.

[8] O'NeILL (B.) .- The fundamental equations of a submersion, Mich. Math. J. 13 (1966), pp. 459-469.

[9] ThORPE (J. A.) .- Some remarks on the Gauss-Bonnet integral, J. Math. Mech. 18 (1969), pp. 779-786. 